\title{
A NEW METHOD TO OBTAIN DECAY RATE ESTIMATES FOR DISSIPATIVE SYSTEMS*
}

\author{
PATRICK MARTINEZ ${ }^{1}$
}

\begin{abstract}
We consider the wave equation damped with a boundary nonlinear velocity feedback $\rho\left(u^{\prime}\right)$. Under some geometrical conditions, we prove that the energy of the system decays to zero with an explicit decay rate estimate even if the function $\rho$ has not a polynomial behavior in zero. This work extends some results of Nakao, Haraux, Zuazua and Komornik, who studied the case where the feedback has a polynomial behavior in zero and completes a result of Lasiecka and Tataru. The proof is based on the construction of a special weight function (that depends on the behavior of the function $\rho$ in zero), and on a new nonlinear integral inequality.
\end{abstract}

Résumé. On considère l'équation des ondes stabilisée par un terme d'amortissement non linéaire $\rho\left(u^{\prime}\right)$ dépendant de la vitesse et agissant sur la frontière. Sous certaines conditions géométriques, on prouve que l'énergie du système décroît vers zéro avec une estimation explicite de décroissance même si la fonction $\rho$ n'a pas un comportement polynomial en zéro. Ce travail étend des résultats de Nakao, Haraux, Zuazua et Komornik, qui ont étudié le cas où le terme d'amortissement a un comportement polynomial en zéro, et complète un résultat de Lasiecka et Tataru. La preuve est basée sur la construction d'une fonction poids spéciale (qui dépend du comportement de la fonction $\rho$ en zéro), et sur une nouvelle inégalité intégrale non linéaire.

AMS Subject Classification. 26A12, 35B40, 93D15.

Received March 26, 1998. Revised October 28, 1998 and April 2, 1999.

\section{INTRODUCTION}

Let $\Omega$ be a bounded open domain of class $\mathcal{C}^{2}$ in $\mathbb{R}^{N}$ and let $\left\{\Gamma_{0}, \Gamma_{1}\right\}$ be a partition of its boundary $\Gamma$. Denote $\nu$ the outward unit normal vector to $\Gamma$. Fix $x_{0}$ in $\mathbb{R}^{N}$ and define

$$
m(x)=x-x_{0} .
$$

Keywords and phrases: Nonlinear stabilization, asymptotic behavior in zero and at infinity.

* This work was done while the author was working in the Institut de Recherche Mathématique Avancée, Université Louis Pasteur Strasbourg I, 7 rue René Descartes, 67084 Strasbourg Cédex, France.

${ }^{1}$ Département de Mathématiques, ENS Cachan, Antenne de Bretagne and IRMAR, Université Rennes I, Campus de Ker Lann, 35170 Bruz, France; e-mail: martinez@bretagne.ens-cachan.fr 
Let $\rho: \mathbb{R} \longrightarrow \mathbb{R}$ be a nondecreasing continuous function such that $\rho(0)=0$. We are concerned with the decay property of the solutions of the problem of the wave equation damped by a nonlinear boundary feedback:

$$
\left\{\begin{array}{l}
u^{\prime \prime}-\Delta u=0 \text { in } \Omega \times \mathbb{R}_{+} \\
u=0 \text { on } \Gamma_{0} \times \mathbb{R}_{+} \\
\partial_{\nu} u+m \cdot \nu \rho\left(u^{\prime}\right)=0 \text { on } \Gamma_{1} \times \mathbb{R}_{+} \\
u(0)=u^{0}, u^{\prime}(0)=u^{1}
\end{array}\right.
$$

As usual, we define the energy of the solution by

$$
E(t)=\frac{1}{2} \int_{\Omega} u^{\prime 2}+|\nabla u|^{2} d x
$$

We assume that there exist a strictly increasing and odd function $g$ of class $\mathcal{C}^{1}$ on $[-1,1]$ and two positive constants $c_{1}$ and $c_{2}$ such that

$$
\left\{\begin{array}{l}
\forall y \in[-1,1],|g(y)| \leq|\rho(y)| \leq\left|g^{-1}(y)\right|, \\
\forall|y| \geq 1, c_{1}|y| \leq|\rho(y)| \leq c_{2}|y|
\end{array}\right.
$$

where $g^{-1}$ denotes the inverse function of $g$.

This problem has been widely studied when

$$
g(y)=c_{3} y^{p} \text { on }[0,1], \text { with some } p>1:
$$

Zuazua [35] proved that the energy decays exponentially if $p=1$ and in a polynomial way if $p>1$ : in this case, there exists some positive constant $C$ such that

$$
\forall t \geq 0, E(t) \leq \frac{C}{(1+t)^{2 /(p-1)}} .
$$

When the function $\rho$ is weaker than any polynomial in zero, for instance if

$$
\forall y \in] 0,1], \rho(y)=e^{-1 / y} .
$$

Lasiecka and Tataru [22] proved that the energy of the solution decays faster than the solution of some associated differential equation.

The goal of this work is to provide an explicit decay estimate of the energy even if $\rho$ has not a polynomial behavior in zero. We prove the following result: under some geometrical assumptions, the energy decays as

$$
\forall t \geq 1, E(t) \leq C\left(g^{-1}\left(\frac{1}{t}\right)\right)^{2}
$$

(see Th. 2). In the example we choose, this formula gives the following estimate:

$$
\forall t \geq 1, E(t) \leq \frac{C}{(\ln (1+t))^{2}} .
$$

The proof is based on the construction of a special weight function $\phi$ and on the generalization of a technique of partition of the boundary introduced by Zuazua. The function $\phi$ and the new partition will be closely related to the behavior of the function $\rho$ in zero (see Sect. 4.2). We need also to generalize a nonlinear integral inequality used by Haraux and Komornik (see Lem. 3). 


\section{StATEMENT OF THE PROBLEM AND MAIN RESUlT}

Let $\Omega$ be a bounded open domain of class $\mathcal{C}^{2}$ in $\mathbb{R}^{N}$ and let $\left\{\Gamma_{0}, \Gamma_{1}\right\}$ be a partition of its boundary $\Gamma$. Denote $\nu$ the outward unit normal vector to $\Gamma$. Fix $x_{0}$ in $\mathbb{R}^{N}$ and define

$$
m(x)=x-x_{0} .
$$

Let $\rho: \mathbb{R} \longrightarrow \mathbb{R}$ be a nondecreasing continuous function such that $\rho(0)=0$. We are concerned with the decay property of the solutions of the following evolutionary problem:

$$
\begin{gathered}
u^{\prime \prime}-\Delta u=0 \text { in } \Omega \times \mathbb{R}_{+}, \\
u=0 \text { on } \Gamma_{0} \times \mathbb{R}_{+}, \\
\partial_{\nu} u+m \cdot \nu \rho\left(u^{\prime}\right)=0 \text { on } \Gamma_{1} \times \mathbb{R}_{+}, \\
u(0)=u^{0}, u^{\prime}(0)=u^{1} .
\end{gathered}
$$

We assume that

$$
\begin{gathered}
\Gamma_{0} \neq \emptyset \text { and } \overline{\Gamma_{0}} \cap \overline{\Gamma_{1}}=\emptyset, \\
m \cdot \nu \leq 0 \text { on } \Gamma_{0} \text { and } m \cdot \nu \geq 0 \text { on } \Gamma_{1} ;
\end{gathered}
$$

(for example, if $\Omega=\Omega_{1} \backslash \Omega_{0}$, where $\Omega_{0}$ and $\Omega_{1}$ are convex sets such that $\overline{\Omega_{0}} \subset \Omega_{1}$, then (2.5-2.6) is satisfied with $\Gamma_{0}=\partial \Omega_{0}, \Gamma_{1}=\partial \Omega_{1}$ and $\left.x_{0} \in \Omega_{0}\right)$.

Moreover we assume that there exist a strictly increasing and odd function $g$ of class $\mathcal{C}^{1}$ on $[-1,1]$ and two positive constants $c_{1}$ and $c_{2}$ such that

$$
\left\{\begin{array}{l}
\forall y \in[-1,1],|g(y)| \leq|\rho(y)| \leq\left|g^{-1}(y)\right| \\
\forall|y| \geq 1, c_{1}|y| \leq|\rho(y)| \leq c_{2}|y|
\end{array}\right.
$$

where $g^{-1}$ denotes the inverse function of $g$. Set

$$
G(y)=y g(y) \text { and } H(y)=\frac{g(y)}{y} .
$$

Note that $H(0)=g^{\prime}(0)$. As usually, denote

$$
H_{\Gamma_{0}}^{1}(\Omega):=\left\{u \in H^{1}(\Omega), u=0 \text { on } \Gamma_{0}\right\} .
$$

The existence and the regularity of the solution $u$ is given by the following standard theorem (see, e.g., Komornik [18] p. 92):

Theorem 1. Assume (2.5-2.6).

1. Given $\left(u^{0}, u^{1}\right) \in H_{\Gamma_{0}}^{1}(\Omega) \times L^{2}(\Omega)$, the problem (2.1-2.4) has a unique solution satisfying

$$
u \in \mathcal{C}\left(\mathbb{R}_{+}, H_{\Gamma_{0}}^{1}(\Omega)\right) \cap \mathcal{C}^{1}\left(\mathbb{R}_{+}, L^{2}(\Omega)\right) .
$$

The energy of the solution $u$ defined by

$$
E(t)=\frac{1}{2} \int_{\Omega} u^{\prime 2}+|\nabla u|^{2} d x
$$

is nonincreasing. 
2. Moreover, if $\rho$ is globally Lipschitz, given $\left(u^{0}, u^{1}\right) \in\left(H^{2}(\Omega) \cap H_{\Gamma_{0}}^{1}(\Omega)\right) \times H_{\Gamma_{0}}^{1}(\Omega)$ satisfying

$$
\partial_{\nu} u^{0}+m \cdot \nu \rho\left(u^{1}\right)=0 \text { on } \Gamma_{1},
$$

the solution of (2.1-2.4) has the stronger regularity property

$$
\begin{gathered}
u \in L^{\infty}\left(\mathbb{R}_{+}, H^{2}(\Omega)\right), \quad u^{\prime} \in L^{\infty}\left(\mathbb{R}_{+}, H_{\Gamma_{0}}^{1}(\Omega)\right), \\
\text { and } u^{\prime \prime} \in L^{\infty}\left(\mathbb{R}_{+}, L^{2}(\Omega)\right) .
\end{gathered}
$$

The problem of the energy decay has been widely studied under different geometrical conditions and different assumptions on the function $\rho$. Several authors derived an explicit decay rate estimate when $\rho$ has a polynomial behavior in zero, that means when

$$
\forall y \in[0,1], g(y)=c_{3} y^{p}, \text { with } p \geq 1:
$$

when $p=1$, the energy decays exponentially to zero: there exist two positive constants $C$ and $\omega$ such that, given $\left(u^{0}, u^{1}\right) \in H_{\Gamma_{0}}^{1}(\Omega) \times L^{2}(\Omega)$, the energy of the solution $u$ satisfies

$$
\forall t \geq 0, E(t) \leq C E(0) e^{-\omega t}
$$

first this estimate has been proved when $\rho$ is linear, i.e. $\rho(y)=\alpha y$ for all $y \in \mathbb{R}$ and some $\alpha>0$, by Chen [5] under suitable geometrical conditions; next Lagnese [20], Komornik and Zuazua [16] extended in various directions Chen's result with the multiplier method and Bardos et al. [3] gave a necessary and sufficient geometrical condition to the exponential decay of the energy using microlocal techniques; when $\rho$ is nonlinear (2.15) has been first proved by Zuazua [35].

When $p>1$, the energy decays at least polynomially to zero: given $\left(u^{0}, u^{1}\right) \in H_{\Gamma_{0}}^{1}(\Omega) \times L^{2}(\Omega)$, there exists a positive constant $C$ that depends on $E(0)$ in a continuous way such that

$$
\forall t \geq 1, E(t) \leq \frac{C}{t^{2 /(p-1)}} .
$$

Such polynomial decay estimates were first obtained on the problem of the wave equation damped by a nonlinear feedback distributed in the domain: Nakao [29] and Haraux [11] studied the case where $\rho(y)=|y|^{p-1} y$ for all $y \in \mathbb{R}$ and some $p>1$. Next Zuazua [34] studied the same problem, but noted that under some suitable growth assumptions on $\rho$, the decay rate was really depending only on the behavior of $\rho$ in zero. Then the problem of stabilization by a nonlinear boundary feedback was studied; Conrad et al. [8], Zuazua [35] and Komornik [18] obtained such polynomial estimates under some different assumptions on the behavior of $\rho$ in zero and with different methods. But all their proofs are closely related to the fact that $\rho$ has a polynomial behavior in zero. We refer the reader to $[1,6,9,21,30,31]$ for related results and to $[4,19]$ for a precise study of the constant $C$ that appears in (2.16).

Note that recently, Vancostenoble [32] proved that this estimate is optimal for a one-dimensional wave equation. More precisely, she studied the following problem

$$
\left\{\begin{array}{l}
u^{\prime \prime}-u_{x x}=0 \text { in }(0,1) \times \mathbb{R}_{+} \\
u(0, t)=0 \text { for all } t \geq 0 \\
u_{x}(1, t)+q\left(u^{\prime}(1, t)\right)=0 \text { for all } t \geq 0 \\
u(0)=u^{0}, u^{\prime}(0)=u^{1}
\end{array}\right.
$$


with $q(y)=y|y|^{p-1}$ in a neighborhood of zero (with $p>1$ ). She proved that there exists a positive constant $C_{p}$ such that, for some initial conditions $\left(u^{0}, u^{1}\right)$, the energy of the solution $u$ satisfies:

$$
E(t) \sim \frac{C_{p}}{t^{2 /(p-1)}} \text { when } t \longrightarrow+\infty
$$

When the function $\rho$ has not a polynomial behavior in zero, there were very few results. Lasiecka and Tataru [22] studied the more general case of a semilinear wave equation damped with a nonlinear velocity feedback acting on $\Gamma_{1}$, under some very weak geometrical conditions on $\Gamma_{0}$ and $\Gamma_{1}$. Without assuming that $\rho$ has a polynomial behavior in zero (and always assuming that $\rho$ has a linear growth at infinity), they proved that the energy decays as fast as the solution of some associated differential equation. More precisely, they generalized the method used to obtain uniform decay estimates when $\rho$ has a polynomial behavior in zero, and they proved that the energy $E(t)$ of the solution $u$ of $(2.1-2.4)$ satisfies

$$
\forall t \geq T_{0}, E(t) \leq S\left(t-T_{0}\right) E(0)
$$

where $S(t)$ is the solution of the following differential equation:

$$
S^{\prime}(t)+q(S(t))=0
$$

where $q$ is a strictly increasing function that can be determined from $\rho$ through some algorithm (what is really important is the behavior of $\rho$ in zero) (see also $[14,15,23,24]$ for similar decay estimates for other problems of nonlinear stabilization). In the particular case where $\rho$ has a polynomial behavior in zero, their result gives again the estimate (2.16); in some specific cases (see in particular Ex. 2 and Ex. 3 in the following), it is possible to obtain a decay rate estimate for the energy; however, it seems quite difficult to obtain a simple decay rate estimate in the general case. Recently, Liu and Zuazua [26] proved the same kind of result with a rather simpler differential equation for the function $S$.

Our goal is to obtain global, uniform and explicit decay rate estimates for the energy when the feedback is not supposed to have a polynomial behavior in zero, in particular when it is weaker than that at zero. This requires the development of new techniques. Our method is based first on special boundary partitions, that depend on the behavior of the feedback in zero, and on some new nonlinear integral inequalities that give precise decay rate estimates. The proof lies on the construction of some special weight function $t \mapsto \phi(t)$, whose growth at infinity is closely related to the behavior of $g$ in zero. Our result is the following

Theorem 2. Assume (2.5-2.6) and (2.7). Then for any given $\left(u^{0}, u^{1}\right) \in H_{\Gamma_{0}}^{1}(\Omega) \times L^{2}(\Omega)$, the solution $u$ of (2.1-2.4) satisfies the estimate

$$
\forall t \geq 1, E(t) \leq C\left(G^{-1}\left(\frac{1}{t}\right)\right)^{2}
$$

with a constant $C$ only depending on the initial energy $E(0)$ (and in a continuous way).

Moreover if $H(0)=0$ and if $H$ is nondecreasing on $[0, \eta]$ for some $\eta>0$, then in fact we have the following better estimate:

$$
\forall t \geq 1, E(t) \leq C\left(g^{-1}\left(\frac{1}{t}\right)\right)^{2}
$$

with a constant $C$ only depending on the initial energy $E(0)$ (and in a continuous way). 
Note that the case where $\rho$ has a polynomial behavior in zero corresponds to the case $g(y)=c y^{p}$ for $y \in[0,1]$. As an illustration, we apply our results to some typical cases:

Example 1. If

$$
g(y)=c y^{p} \leq \rho(y) \leq g^{-1}(y)=c^{\prime} y^{1 / p} \text { if } 0<y<1
$$

for some $p>1$, then (2.20) gives that

$$
\forall t \geq 0, E(t) \leq C(1+t)^{-2 / p}
$$

which is a less good estimate than (2.16). We did not manage to find directly the estimate (2.16) with our method; however it can be proved that, given $\varepsilon>0$, there exists a positive constant $C_{p}$ that does not depend on $\varepsilon$ such that:

$$
\forall t \geq 0, E(t) \leq C_{p}(1+t)^{\frac{-2}{p-1}+\varepsilon}
$$

Letting go $\varepsilon$ to zero, we find the estimate (2.16) (see Sect. 4.4).

We can apply also our result to other more general cases:

Example 2. If

$$
g(y)=e^{-1 / y^{p}} \leq \rho(y) \leq g^{-1}(y)=\left(\frac{-1}{\ln y}\right)^{1 / p} \text { if } 0<y<1
$$

for some $p>0$, then (2.20) gives the estimate

$$
\forall t \geq 2, E(t) \leq \frac{C}{(\ln t)^{2 / p}} .
$$

Example 3. If

$$
g(y)=e^{-e^{1 / y}} \leq \rho(y) \leq g^{-1}(y)=\frac{1}{\ln (-\ln y)} \text { if } 0<y<1,
$$

then (2.20) gives the estimate

$$
\forall t \geq 3, E(t) \leq \frac{C}{(\ln (\ln t))^{2}}
$$

Remarks. 1. In Examples 1, 2 and 3, we find directly the same estimate than the one provided by the results of Lasiecka and Tataru [22] and of Liu and Zuazua [26]. In a recent work with Vancostenoble, we proved that these last estimates are in fact optimal for the problem (2.17). More generally, we proved that the decay rate estimate provided by (2.20) is optimal for a class of functions $g$ including Example 2 and Example 3 (see [33] for several optimality results).

2. We present our results under the geometrical hypothesis $(2.5-2.6)$. We shall treat exactly in the same way the following problem

$$
\left\{\begin{array}{l}
u^{\prime \prime}-\Delta u+q u=0 \text { in } \Omega \times \mathbb{R}_{+} \\
\partial_{\nu} u+a(x) m \cdot \nu u+\ell(x) m \cdot \nu \rho\left(u^{\prime}\right)=0 \text { on } \partial \Omega \times \mathbb{R}_{+} \\
u(0)=u^{0}, u^{\prime}(0)=u^{1}
\end{array}\right.
$$


where $q, a$ and $\ell$ are continuous nonnegative functions, provided that $q$ and $a$ are not both equal to zero, and that

$$
m \cdot \nu \geq 0 \text { on } \partial \Omega
$$

using a method introduced by Conrad and Rao [9].

3. The decay estimates are the same for the problem of stabilization of the wave equation damped by a nonlinear localized feedback under suitable assumptions on the localization and the same conditions on the nonlinearity than in the boundary case (see [28]).

4. The method we present here can be applied to study the problem of the wave equation damped by a linear localized feedback $a(x) u^{\prime}$ when the function $a$ goes quickly to zero at the boundary (see [27]).

In Section 3, we state several new nonlinear integral inequalities that generalize results of Haraux [12] and Komornik [18]. The goal of this section is to prove Lemma 3, which contains the inequality we need to prove Theorem 2. In Section 4 we prove Theorem 2 when $\rho$ is globally Lipschitz: first we prove Lemma 6 by the multiplier method; then we construct explicitly special boundary partitions of $\Gamma_{1}$ and a weight function $\phi$ that allow us to apply Lemma 3 and to prove the estimates (2.19) and (2.20). In Section 5, we complete the proof of Theorem 2 removing the additional hypothesis on $\rho$.

\section{Some NONLINEAR INTEGRAL INEQUALITIES}

First we prove some intermediate results that are useful to get Lemma 3:

\subsection{The key integral inequality}

The following results of this section are based on the following integral inequality:

Lemma 1. Let $E: \mathbb{R}_{+} \longrightarrow \mathbb{R}_{+}$be a nonincreasing function and $\phi: \mathbb{R}_{+} \longrightarrow \mathbb{R}_{+}$a strictly increasing function of class $\mathcal{C}^{1}$ such that

$$
\phi(0)=0 \text { and } \phi(t) \longrightarrow+\infty \text { as } t \longrightarrow+\infty .
$$

Assume that there exist $\sigma \geq 0$ and $\omega>0$ such that:

$$
\forall S \geq 0, \int_{S}^{+\infty} E(t)^{1+\sigma} \phi^{\prime}(t) d t \leq \frac{1}{\omega} E(0)^{\sigma} E(S) .
$$

Then $E$ has the following decay property:

$$
\begin{gathered}
\text { if } \sigma=0, \text { then } E(t) \leq E(0) e^{1-\omega \phi(t)}, \forall t \geq 0, \\
\text { if } \sigma>0 \text {, then } E(t) \leq E(0)\left(\frac{1+\sigma}{1+\omega \sigma \phi(t)}\right)^{1 / \sigma}, \forall t \geq 0 .
\end{gathered}
$$

Remark. Haraux [11,12] and Komornik [18] proved such results when $\phi(t)=t$ for all $t \geq 0$. Their results can only be applied when $E$ decays to zero at least in a polynomial way. The novelty of Lemma 1 comes from the introduction of the weight function $\phi^{\prime}(t)$, that allows us to consider functions $E$ that can decay slowly to zero. For example, if $E$ verifies (3.2) with

$$
\phi(t)=\ln (\ln (3+t))-\ln (\ln 3) \text { and } \sigma=0,
$$


or with

$$
\phi(t)=\ln (3+t)-\ln 3 \text { and } \sigma>0,
$$

then we deduce that

$$
\forall t \geq 0, E(t) \leq \frac{c E(0)}{(\ln (3+t))^{\gamma}}
$$

with $\gamma=\omega$ if $\sigma=0$ and $\gamma=1 / \sigma$ if $\sigma>0$.

Note that if $\phi(0) \neq 0$, it is sufficient to replace $\phi(t)$ by $\phi(t)-\phi(0)$ in (3.3) and (3.4).

Proof of Lemma 1. Let us introduce $f:\left[0,+\infty\left[\longrightarrow \mathbb{R}_{+}\right.\right.$defined by

$$
f(\tau)=E\left(\phi^{-1}(\tau)\right)
$$

Then $f$ is nonincreasing and satisfies

$$
\begin{aligned}
\forall 0 \leq S<T<\infty: \int_{\phi(S)}^{\phi(T)} f(\tau)^{1+\sigma} d \tau & =\int_{\phi(S)}^{\phi(T)} E\left(\phi^{-1}(\tau)\right)^{1+\sigma} d \tau \\
& =\int_{S}^{T} E(t)^{1+\sigma} \phi^{\prime}(t) d t \leq \frac{1}{\omega} E(0)^{\sigma} E(S)=\frac{1}{\omega} f(0)^{\sigma} f(\phi(S)) .
\end{aligned}
$$

Denote $s=\phi(S)$. As $\lim _{T \rightarrow+\infty} \phi(T)=+\infty, f$ satisfies:

$$
\forall s \geq 0, \int_{s}^{+\infty} f(\tau)^{1+\sigma} d \tau \leq \frac{1}{\omega} f(s) .
$$

Then a well-known Gronwall type result (see Komornik [18] p. 124) gives us that

$$
\text { if } \sigma=0, f(s) \leq f(0) e^{1-\omega s}, \forall s \geq 0,
$$

and

$$
\text { if } \sigma>0, f(s) \leq f(0)\left(\frac{1+\sigma}{1+\omega \sigma s}\right)^{1 / \sigma} \forall s \geq 0 .
$$

Since $E(t)=f(\phi(t)),(3.3)$ and (3.4) follow.

\subsection{Consequences}

First we deduce from Lemma 1 the following result:

Corollary 3.1. Let $f: \mathbb{R}_{+} \longrightarrow \mathbb{R}_{+}$be a nonincreasing continuous function.

Assume that there exist $\sigma>0, \sigma^{\prime} \geq 0$ and $c>0$ such that:

$$
\forall t \geq 0, \int_{t}^{+\infty} f(\tau)^{1+\sigma} d \tau \leq c \frac{f(0)^{\sigma} f(t)}{(1+t)^{\sigma^{\prime}}} .
$$

Then there exists $C>0$ such that:

$$
\forall t \geq 0, f(t) \leq f(0) \frac{C}{(1+t)^{\left(1+\sigma^{\prime}\right) / \sigma}} .
$$


Proof of Corollary 3.1. We can assume that $f(0)=1$. Define

$$
g(t)=\frac{f(t)}{(1+t)^{\sigma^{\prime}}} \text { if } t \geq 0 .
$$

$g$ is nonincreasing and satisfies

$$
\forall t \geq 0, \int_{t}^{+\infty} g(\tau)^{1+\sigma}(1+\tau)^{\sigma^{\prime}(1+\sigma)} d \tau \leq c g(t) .
$$

Then we can apply Lemma 1 with

$$
\phi(t)=(1+t)^{\sigma^{\prime}(1+\sigma)+1}-1
$$

to deduce that $g$ decays as

$$
g(t) \leq \frac{C}{(1+t)^{\left(\sigma^{\prime}(1+\sigma)+1\right) / \sigma}}=\frac{C}{(1+t)^{\sigma^{\prime}}(1+t)^{\left(1+\sigma^{\prime}\right) / \sigma}} .
$$

So (3.6) follows.

Then we deduce the following integral inequality from Corollary 3.1:

Lemma 2. Let $f: \mathbb{R}_{+} \longrightarrow \mathbb{R}_{+}$be a nonincreasing continuous function. Assume that there exist $\sigma>0, \sigma^{\prime} \geq 0$ and $c>0$ such that:

$$
\forall t \geq 0, \int_{t}^{+\infty} f(\tau)^{1+\sigma} d \tau \leq c f(t)^{1+\sigma}+\frac{c}{(1+t)^{\sigma^{\prime}}} f(0)^{\sigma} f(t) .
$$

Then there exists $C>0$ such that

$$
\forall t \geq 0, f(t) \leq f(0) \frac{C}{(1+t)^{\left(1+\sigma^{\prime}\right) / \sigma}}
$$

Proof of Lemma 2. We can assume that $f(0)=1$. The idea is that the influence of the term $f(t)^{1+\sigma}$ on the decay estimate of $f$ is negligible with respect to the influence of the second term: when we have only the term $f(t)^{1+\sigma}$ on the right-hand side of (3.7), we know that $f$ decays exponentially to zero, but when we have only the second term, we know that $f$ decays at least polynomially to zero. We prove (3.8) using an induction argument. In the following we denote by $C$ all the positive constants.

First we deduce from (3.7) that

$$
\forall t \geq 0, \int_{t}^{+\infty} f(\tau)^{1+\sigma} d \tau \leq C f(t)
$$

So we deduce from Corollary 3.1

$$
\forall t \geq 0, f(t) \leq \frac{C}{(1+t)^{1 / \sigma}} .
$$

Then we use this estimate in (3.7) to deduce that $f$ satisfies

$$
\forall t \geq 0, \int_{t}^{+\infty} f(\tau)^{1+\sigma} d \tau \leq C \frac{f(t)}{(1+t)}+C \frac{f(t)}{(1+t)^{\sigma^{\prime}}} .
$$


Define $\sigma_{1}=\inf \left\{1, \sigma^{\prime}\right\}$. Then we have

$$
\forall t \geq 0, \int_{t}^{+\infty} f(\tau)^{1+\sigma} d \tau \leq C \frac{f(t)}{(1+t)^{\sigma_{1}}}
$$

and we conclude using (3.6) that

$$
\forall t \geq 0, f(t) \leq \frac{C}{(1+t)^{\left(1+\sigma_{1}\right) / \sigma}}
$$

If $\sigma^{\prime} \leq 1$ we get (3.8). In the contrary

$$
\forall t \geq 0, f(t) \leq \frac{C}{(1+t)^{2 / \sigma}}
$$

Set $n \in \mathbb{N}$ such that $\sigma \in[n, n+1]$. We prove by induction that for all $k \in \mathbb{N}, k \leq n, f$ satisfies:

$$
\forall t \geq 0, f(t) \leq \frac{C_{k}}{(1+t)^{(1+k) / \sigma}} .
$$

We have proved (3.9) for $k=0$ and for $k=1$ if $n \geq 1$. Assume that $n \geq 2$ and that (3.9) is true for some $k<n$. Then we use (3.9) to deduce from (3.7) that $f$ satisfies:

$$
\forall t \geq 0, \int_{t}^{+\infty} f(\tau)^{1+\sigma} d \tau \leq C \frac{f(t)}{(1+t)^{1+k}}+C \frac{f(t)}{(1+t)^{\sigma^{\prime}}} .
$$

Since $1+k \leq n \leq \sigma^{\prime}$, we have

$$
\forall t \geq 0, \int_{t}^{+\infty} f(\tau)^{1+\sigma} d \tau \leq C \frac{f(t)}{(1+t)^{1+k}},
$$

and we deduce from Corollary 3.1 that

$$
\forall t \geq 0, f(t) \leq \frac{C_{k}}{(1+t)^{(2+k) / \sigma}}
$$

That shows that

$$
\forall t \geq 0, f(t) \leq \frac{C_{k}}{(1+t)^{(n+1) / \sigma}}
$$

and then with the same argument:

$$
\forall t \geq 0, f(t) \leq \frac{C_{k}}{(1+t)^{\left(\sigma^{\prime}+1\right) / \sigma}}
$$

Now we can state the integral inequality that we will use to prove Theorem 2:

Lemma 3. Let $E: \mathbb{R}_{+} \longrightarrow \mathbb{R}_{+}$be a nonincreasing function and $\phi: \mathbb{R}_{+} \longrightarrow \mathbb{R}_{+}$a strictly increasing function of class $\mathcal{C}^{1}$ such that

$$
\phi(0)=0 \text { and } \phi(t) \longrightarrow+\infty \text { as } t \longrightarrow+\infty .
$$


Assume that there exist $\sigma>0, \sigma^{\prime} \geq 0$ and $c>0$ such that:

$$
\forall S \geq 0, \int_{S}^{+\infty} E(t)^{1+\sigma} \phi^{\prime}(t) d t \leq c E(S)^{1+\sigma}+\frac{c}{(1+\phi(S))^{\sigma^{\prime}}} E(0)^{\sigma} E(S) .
$$

Then there exists $C>0$ such that

$$
\forall t>0, E(t) \leq E(0) \frac{C}{(1+\phi(t))^{\left(1+\sigma^{\prime}\right) / \sigma}}
$$

Proof of Lemma 3. It is sufficient to introduce

$$
f(\tau)=E\left(\phi^{-1}(\tau)\right)
$$

and to use Lemma 2.

\section{Proof of Theorem 2 When $\rho$ IS Globally Lipschitz}

In this section, we prove Theorem 2 under the following additional hypothesis:

$$
\rho \text { is globally Lipschitz. }
$$

This hypothesis will be removed in the last section.

We note that it is sufficient to prove (2.19) (or (2.20)) for initial conditions $\left(u^{0}, u^{1}\right) \in\left(H^{2}(\Omega) \cap H_{\Gamma_{0}}^{1}(\Omega)\right)$ $\times H_{\Gamma_{0}}^{1}(\Omega)$ satisfying (2.11). Then a standard density argument gives (2.19) (or (2.20)) for all initial condition in $H_{\Gamma_{0}}^{1}(\Omega) \times L^{2}(\Omega)$. Set $\left(u^{0}, u^{1}\right) \in\left(H^{2}(\Omega) \cap H_{\Gamma_{0}}^{1}(\Omega)\right) \times H_{\Gamma_{0}}^{1}(\Omega)$ satisfying (2.11). The regularity of the solution $u$ of $(2.1-2.4)$ given by $(2.12-2.13)$ justifies all the following computations (where we will omit to write the differential elements).

\subsection{The key identity given by the multiplier method}

First we need an expression of $E^{\prime}$ :

Lemma 4. The function $E: \mathbb{R}_{+} \longrightarrow \mathbb{R}_{+}$is nonincreasing, locally absolutely continuous and

$$
E^{\prime}(t)=-\int_{\Gamma_{1}} m \cdot \nu u^{\prime} \rho\left(u^{\prime}\right) d s
$$

Proof of Lemma 4. This is a well known result. We multiply (2.1) by $u^{\prime}$ and we integrate by parts on $\Omega \times[S, T]$ :

$$
0=\int_{S}^{T} \int_{\Omega} u^{\prime}\left(u^{\prime \prime}-\Delta u\right)=\left[\frac{1}{2} \int_{\Omega} u^{\prime 2}+|\nabla u|^{2}\right]_{S}^{T}-\int_{S}^{T} \int_{\Gamma} u^{\prime} \partial_{\nu} u=E(T)-E(S)+\int_{S}^{T} \int_{\Gamma} m \cdot \nu u^{\prime} \rho\left(u^{\prime}\right) .
$$

The proof of Theorem 2 is based on the following identity given by the multiplier method:

Lemma 5. Let $\phi: \mathbb{R}_{+} \longrightarrow \mathbb{R}$ a function of class $\mathcal{C}^{2}$. Set $0 \leq S<T<+\infty$. Putting for brevity

$$
M(u)=2 m \cdot \nabla u+(N-1) u
$$


we have

$$
\begin{aligned}
2 \int_{S}^{T} E^{2} \phi^{\prime} d t & =\int_{S}^{T} E \phi^{\prime} \int_{\Gamma} M(u) \partial_{\nu} u+m \cdot \nu\left(u^{\prime 2}-|\nabla u|^{2}\right) d s d t \\
& +\int_{S}^{T}\left(E^{\prime} \phi^{\prime}+E \phi^{\prime \prime}\right) \int_{\Omega} u^{\prime} M(u) d x d t-\left[E \phi^{\prime} \int_{\Omega} u^{\prime} M(u) d x\right]_{S}^{T} .
\end{aligned}
$$

Remark. This identity is classical when $\phi(t)=t$ for all $t \geq 0$ (see, e.g. Komornik [18] p. 128). The function $\phi$ will be chosen later ( $\phi$ will be closely related to $g$ ).

Proof of Lemma 5. We integrate by parts the expression

$$
\begin{aligned}
0= & \int_{S}^{T} E(t) \phi^{\prime}(t)\left(\int_{\Omega} M(u)\left(u^{\prime \prime}-\Delta u\right) d x\right) d t \\
0= & {\left[E \phi^{\prime} \int_{\Omega} u^{\prime} M(u)\right]_{S}^{T}-\int_{S}^{T}\left(E^{\prime} \phi^{\prime}+E \phi^{\prime \prime}\right) \int_{\Omega} u^{\prime} M(u)-\int_{S}^{T} E \phi^{\prime} \int_{\Omega} 2 u^{\prime} m \cdot \nabla u^{\prime}+(N-1) u^{\prime 2} } \\
& \quad-\int_{S}^{T} E \phi^{\prime} \int_{\Gamma} M(u) \partial_{\nu} u+\int_{S}^{T} E \phi^{\prime} \int_{\Omega} \nabla u \cdot \nabla(2 m \cdot \nabla u)+(N-1)|\nabla u|^{2} \\
= & {\left[E \phi^{\prime} \int_{\Omega} u^{\prime} M(u)\right]_{S}^{T}-\int_{S}^{T}\left(E^{\prime} \phi^{\prime}+E \phi^{\prime \prime}\right) \int_{\Omega} u^{\prime} M(u) } \\
& \quad-\int_{S}^{T} E \phi^{\prime} \int_{\Gamma} m \cdot \nu u^{\prime 2}+\int_{S}^{T} E \phi^{\prime} \int_{\Omega}(\operatorname{div} m) u^{\prime 2}-(N-1) u^{\prime 2} \\
& \quad-\int_{S}^{T} E \phi^{\prime} \int_{\Gamma} M(u) \partial_{\nu} u+\int_{S}^{T} E \phi^{\prime} \int_{\Omega} m \cdot \nabla\left(|\nabla u|^{2}\right)+(N+1)|\nabla u|^{2} \\
= & \left.E \phi^{\prime} \int_{\Omega} u^{\prime} M(u)\right]_{S}^{T}-\int_{S}^{T}\left(E^{\prime} \phi^{\prime}+E \phi^{\prime \prime}\right) \int_{\Omega} u^{\prime} M(u)-\int_{S}^{T} E \phi^{\prime} \int_{\Gamma} M(u) \partial_{\nu} u+m \cdot \nu\left(u^{\prime 2}-|\nabla u|^{2}\right) \\
& +\int_{S}^{T} E \phi^{\prime} \int_{\Omega} u^{\prime 2}+|\nabla u|^{2} .
\end{aligned}
$$

Now assume that $\phi$ is a strictly increasing concave function. So $\phi^{\prime}$ is a bounded function on $\mathbb{R}_{+}$. Denote $\lambda$ the maximum of $\phi^{\prime}$. We deduce from (4.3) the following estimate:

Lemma 6. There exists a positive constant $c$ such that for all $0 \leq S<T$ :

$$
\int_{S}^{T} E(t)^{2} \phi^{\prime}(t) d t \leq c E(S)^{2}+c \int_{S}^{T} E(t) \phi^{\prime}(t)\left(\int_{\Gamma_{1}} m \cdot \nu\left(u^{\prime 2}+\rho\left(u^{\prime}\right)^{2}\right) d s\right) d t .
$$

Proof of Lemma 6. It is easy to show that there exists $c>0$ such that

$$
\left|\int_{\Omega} u^{\prime} M(u)\right| \leq c E(t) .
$$

So

$$
\left|E(t) \phi^{\prime}(t) \int_{\Omega} u^{\prime} M(u)\right| \leq c \lambda E(t)^{2} .
$$


Since $E$ is nonincreasing and $\phi$ is concave:

$$
\left|\int_{S}^{T} E^{\prime} \phi^{\prime} \int_{\Omega} u^{\prime} M(u)\right| \leq c \lambda \int_{S}^{T}-E^{\prime}(t) E(t) d t \leq \frac{c \lambda}{2} E(S)^{2},
$$

and

$$
\left|\int_{S}^{T} E \phi^{\prime \prime} \int_{\Omega} u^{\prime} M(u)\right| \leq c \int_{S}^{T} E(t)^{2}\left(-\phi^{\prime \prime}(t)\right) d t \leq c E(S)^{2} \phi^{\prime}(S) .
$$

Next we look at the boundary integrals: we see that

$$
\int_{S}^{T} E \phi^{\prime} \int_{\Gamma_{0}} \partial_{\nu} u M(u)+m \cdot \nu\left(u^{\prime 2}-|\nabla u|^{2}\right)=\int_{S}^{T} E \phi^{\prime} \int_{\Gamma_{0}} m \cdot \nu\left(\partial_{\nu} u\right)^{2} \leq 0 .
$$

Set $\varepsilon>0$ :

$$
\begin{aligned}
& \int_{S}^{T} E \phi^{\prime} \int_{\Gamma_{1}} \partial_{\nu} u M(u)+m \cdot \nu\left(u^{\prime 2}-|\nabla u|^{2}\right) \\
& =\int_{S}^{T} E \phi^{\prime} \int_{\Gamma_{1}} m \cdot \nu\left(-2 \rho\left(u^{\prime}\right) m \cdot \nabla u-(N-1) u \rho\left(u^{\prime}\right)+u^{\prime 2}-|\nabla u|^{2}\right) \\
& \leq \frac{c}{\varepsilon} \int_{S}^{T} E \phi^{\prime} \int_{\Gamma_{1}} m \cdot \nu\left(u^{\prime 2}+\rho\left(u^{\prime}\right)^{2}\right)+\varepsilon \int_{S}^{T} E \phi^{\prime} \int_{\Gamma_{1}} u^{2} \\
& \leq c^{\prime} \int_{S}^{T} E \phi^{\prime} \int_{\Gamma_{1}} m \cdot \nu\left(u^{\prime 2}+\rho\left(u^{\prime}\right)^{2}\right)+\int_{S}^{T} E^{2} \phi^{\prime}
\end{aligned}
$$

if $\varepsilon$ is small enough. Then the estimate (4.4) follows from (4.3) using (4.6-4.10).

\subsection{First estimate on the decay rate of the energy}

When $\rho$ has a polynomial behavior in zero, the last term of (4.4) can be estimated using first Jensen inequality and then Hölder inequality (see, e.g., Komornik [18] p. 130). But when $\rho$ is weaker than any polynomial in zero, it is more difficult to apply the same method. Lasiecka and Tataru [22] generalized it but did not obtain an explicit decay rate estimate for the energy.

The major problem is to find how the decrease of the energy at infinity is related to the behavior of $\rho$ in zero. The information we need on the behavior of $\rho$ in zero will be contained in the behavior of the weight function $\phi$ at infinity. However we need to construct a suitable function $\phi$ so that we can apply the results of Lemma 1 or Lemma 3. In order to see how $\phi$ will depend on $\rho$, we introduce special partitions of the boundary $\Gamma_{1}$ that will depend on the behavior of $g$ in zero.

It is convenient to construct a strictly increasing odd function $\tilde{g}: \mathbb{R} \longrightarrow \mathbb{R}$ of class $\mathcal{C}^{1}$ on $\mathbb{R}$ such that

$$
\left\{\begin{array}{l}
\forall y \in\left[-\frac{3}{2}, \frac{3}{2}\right], \tilde{g}(y)=g(y) \\
\tilde{g}(1)<1, \\
\forall y \in]-\infty,-1[\cup] 1,+\infty[, \tilde{g}(y)=c y \\
\forall y \in \mathbb{R},|\tilde{g}(y)| \leq|\rho(y)| \leq\left|\tilde{g}^{-1}(y)\right|
\end{array}\right.
$$


with $c:=\inf \left\{c_{1}, \frac{1}{c_{2}}\right\}$. This can be done thanks to $(2.7)$ (note that if $g(1)=1$ and $g^{\prime}(1)>1$, we can not impose on $\tilde{g}$ to satisfy $\tilde{g}=g$ on $[-1,1]$ and $|\tilde{g}(y)| \leq\left|\tilde{g}^{-1}(y)\right|$ on $\mathbb{R}$ if $\tilde{g}$ is of class $\mathcal{C}^{1}$ on $\left.\mathbb{R}\right)$. In the following, since we will be interested in the behavior of $g$ in zero, we still denote $\tilde{g}=g$.

Assume now that $\phi$ is a strictly increasing concave function of class $\mathcal{C}^{2}$ on $[0,+\infty[$ such that

$$
\phi(t) \longrightarrow+\infty \text { and } \phi^{\prime}(t) \longrightarrow 0 \text { as } t \longrightarrow+\infty
$$

(for example $t \mapsto \ln (1+t)$ ). Let us introduce

$$
\forall t \geq 1, h(t)=g^{-1}\left(\phi^{\prime}(t)\right)
$$

$h$ is a decreasing positive function and satisfies

$$
h(t) \longrightarrow 0 \text { as } t \longrightarrow+\infty .
$$

For every $t \geq 1$ define

$$
\begin{aligned}
& \Gamma_{1,1}^{t}:=\left\{x \in \Gamma_{1}:\left|u^{\prime}\right| \leq h(t)\right\} \\
& \Gamma_{1,2}^{t}:=\left\{x \in \Gamma_{1}: h(t)<\left|u^{\prime}\right| \leq h(1)\right\} \\
& \Gamma_{1,3}^{t}:=\left\{x \in \Gamma_{1}:\left|u^{\prime}\right|>h(1)\right\}
\end{aligned}
$$

Thanks to this partition of $\Gamma_{1}$ we prove the following

Lemma 7. There exists a positive constant $c$ such that:

$$
\forall 1 \leq S<T<\infty: \int_{S}^{T} E \phi^{\prime} \int_{\Gamma_{1}} m \cdot \nu{u^{\prime}}^{2} d s d t \leq c E(S)^{2}+c E(S) \int_{S}^{T} \phi^{\prime}(t)\left(g^{-1}\left(\phi^{\prime}(t)\right)\right)^{2} d t .
$$

Proof of Lemma \%. We estimate the integral on each $\Gamma_{1, i}^{t}$ :

Estimate of the part on $\Gamma_{1,3}^{t}$ : since $h(1)>0$, we note that

$$
|\rho(y)| \geq c^{\prime}|y| \text { if }|y| \geq h(1):
$$

the function $H: y \mapsto \frac{\rho(y)}{y}$ is continuous on $\left.]-\infty,-h(1)\right] \cup\left[h(1),+\infty\left[\right.\right.$ and bounded from below by $c_{1}>0$ on ]$-\infty,-1] \cup[1,+\infty[$ thanks to the hypothesis $(2.7)$. So if $h(1) \geq 1,(4.17)$ is satisfied, and if $h(1)<1$, it is sufficient to note that $H$ is positive and continuous on $[-1,-h(1)] \cup[h(1), 1]$, so also bounded from below by $c_{1}^{\prime}$ on $[h(1), 1]$. Then $(4.17)$ is satisfied with $c^{\prime}=\inf \left(c_{1}, c_{1}^{\prime}\right)$.

Then we have

$$
\int_{S}^{T} E \phi^{\prime} \int_{\Gamma_{1,3}^{t}} m \cdot \nu u^{\prime 2} d s d t \leq \frac{1}{c^{\prime}} \int_{S}^{T} E \phi^{\prime} \int_{\Gamma_{1,3}^{t}} m \cdot \nu u^{\prime} \rho\left(u^{\prime}\right) d s d t \leq \frac{\lambda}{c^{\prime}} \int_{S}^{T} E\left(-E^{\prime}\right) d t \leq \frac{\lambda}{2 c^{\prime}} E(S)^{2} .
$$

Estimate of the part on $\Gamma_{1,2}^{t}$ : since $g$ is increasing, if $x \in \Gamma_{1,2}^{t}$, then

$$
\phi^{\prime}(t)=g(h(t)) \leq\left|g\left(u^{\prime}\right)\right|
$$


Thus

$$
\begin{aligned}
\int_{S}^{T} E \phi^{\prime} \int_{\Gamma_{1,2}^{t}} m \cdot \nu u^{\prime 2} d s d t & \leq \int_{S}^{T} E \int_{\Gamma_{1,2}^{t}} m \cdot \nu\left|g\left(u^{\prime}\right)\right| u^{\prime 2} d s d t \\
& \leq h(1) \int_{S}^{T} E \int_{\Gamma_{1,2}^{t}} m \cdot \nu u^{\prime} g\left(u^{\prime}\right) d s d t \\
& \leq h(1) \int_{S}^{T} E \int_{\Gamma_{1,2}^{t}} m \cdot \nu u^{\prime} \rho\left(u^{\prime}\right) d s d t \leq \frac{h(1)}{2} E(S)^{2}
\end{aligned}
$$

Estimate of the part on $\Gamma_{1,1}^{t}$ : thanks to the definition of this part of the boundary, we have:

$$
\int_{S}^{T} E \phi^{\prime} \int_{\Gamma_{1,1}^{t}} m \cdot \nu u^{\prime 2} d s d t \leq c \int_{S}^{T} E(t) \phi^{\prime}(t)\left(\int_{\Gamma_{1,1}^{t}} h(t)^{2} d s\right) d t \leq c|\Gamma| E(S) \int_{S}^{T} \phi^{\prime}(t)\left(g^{-1}\left(\phi^{\prime}(t)\right)\right)^{2} d t
$$

we deduce (4.16) from (4.18-4.20).

Next we estimate the term

$$
\int_{S}^{T} E \phi^{\prime} \int_{\Gamma_{1}} m \cdot \nu \rho\left(u^{\prime}\right)^{2} d s d t
$$

of the right-hand side of (4.4) in the same way. In this section, $\rho$ is supposed to be globally Lipschitz. So, since

$$
\forall y \in \mathbb{R},|\rho(y)| \leq K|y|
$$

where $K$ is the constant of Lipschitz of $\rho$, we could say that

$$
\int_{S}^{T} E \phi^{\prime} \int_{\Gamma_{1}} m \cdot \nu \rho\left(u^{\prime}\right)^{2} d s d t \leq K^{2} \int_{S}^{T} E \phi^{\prime} \int_{\Gamma_{1}} m \cdot \nu u^{\prime 2} d s d t
$$

and then we could use the estimate provided by Lemma 7 . However, this way to estimate is not convenient to eliminate the assumption " $\rho$ is globally Lipschitz": this can be done approximating $\rho$ by a sequence of globally Lipschitz functions $\rho_{k}$ that converges to $\rho$ (see in Sect. 4). So we need to find estimates on the decay rate of the energy of the solution $u_{k}$ (related to the problem $\left(2.1-2.4\right.$ ) where $\rho$ is replaced by $\rho_{k}$ ) that do not depend on the constant of Lipschitz of each function $\rho_{k}$. Using another partition of $\Gamma_{1}$, we prove the following:

Lemma 8. There exists a positive constant $c$ that does not depend on the constant of Lipschitz of $\rho$ such that:

$$
\forall 1 \leq S<T<\infty: \int_{S}^{T} E \phi^{\prime} \int_{\Gamma_{1}} m \cdot \nu \rho\left(u^{\prime}\right)^{2} d s d t \leq c E(S)^{2}+c E(S) \int_{S}^{T} \phi^{\prime}(t)\left(g^{-1}\left(\phi^{\prime}(t)\right)\right)^{2} d t
$$

Proof of Lemma 8. For every $t \geq 1$, we define

$$
\begin{aligned}
& \Gamma_{1,4}^{t}:=\left\{x \in \Gamma_{1}:\left|u^{\prime}\right| \leq \phi^{\prime}(t)\right\} \\
& \Gamma_{1,5}^{t}:=\left\{x \in \Gamma_{1}: \phi^{\prime}(t)<\left|u^{\prime}\right| \leq \phi^{\prime}(1)\right\} \\
& \Gamma_{1,6}^{t}:=\left\{x \in \Gamma_{1}:\left|u^{\prime}\right|>\phi^{\prime}(1)\right\}
\end{aligned}
$$


With this partition of $\Gamma_{1}$ we easily see that

$$
\int_{S}^{T} E \phi^{\prime} \int_{\Gamma_{1,6}^{t}} m \cdot \nu \rho\left(u^{\prime}\right)^{2} d s d t \leq c \int_{S}^{T} E \phi^{\prime} \int_{\Omega_{1,6}^{t}} m \cdot \nu u^{\prime} \rho\left(u^{\prime}\right) d s d t \leq c \lambda \int_{S}^{T} E\left(-E^{\prime}\right) d t \leq c \lambda E(S)^{2} .
$$

Next we look at the part on $\Gamma_{1,5}^{t}$ : by monotonicity, if $x \in \Gamma_{1,5}^{t}$, then

$$
\begin{aligned}
\int_{S}^{T} E \phi^{\prime} \int_{\Gamma_{1,5}^{t}} m \cdot \nu \rho\left(u^{\prime}\right)^{2} d s d t & \leq \int_{S}^{T} E \int_{\Gamma_{1,5}^{t}} m \cdot \nu\left|u^{\prime}\right| \rho\left(u^{\prime}\right)^{2} d s d t \\
& \leq c \int_{S}^{T} E \int_{\Gamma_{1,5}^{t}} m \cdot \nu u^{\prime} \rho\left(u^{\prime}\right) d s d t \leq c E(S)^{2}
\end{aligned}
$$

At last we look at the part on $\Gamma_{1,4}^{t}$ :

$$
\begin{aligned}
\int_{S}^{T} E \phi^{\prime} \int_{\Gamma_{1,4}^{t}} m \cdot \nu \rho\left(u^{\prime}\right)^{2} d s d t & \leq c \int_{S}^{T} E(t) \phi^{\prime}(t)\left(\int_{\Gamma_{1,4}^{t}}\left(g^{-1}\left(\left|u^{\prime}\right|\right)\right)^{2} d s\right) d t \\
& \leq c|\Gamma| E(S) \int_{S}^{T} \phi^{\prime}(t)\left(g^{-1}\left(\phi^{\prime}(t)\right)\right)^{2} d t .
\end{aligned}
$$

We deduce (4.21) from (4.25-4.27).

Now assume that $\phi$ satisfies the following additional property:

$$
\int_{1}^{\infty} \phi^{\prime}(t)\left(g^{-1}\left(\phi^{\prime}(t)\right)\right)^{2} d t \text { converges. }
$$

This property is closely related to the behavior of $g$ near 0 and the decay rate of $\phi^{\prime}$ at infinity. Then we deduce from (4.4) and the estimates (4.16) and (4.21) that there exists a positive constant $c$ such that

$$
\forall 1 \leq S<T, \int_{S}^{T} E(t)^{2} \phi^{\prime}(t) d t \leq c E(S)^{2}+c E(S) \int_{S}^{+\infty} \phi^{\prime}(t)\left(g^{-1}\left(\phi^{\prime}(t)\right)\right)^{2} d t
$$

In particular that implies that

$$
\forall S \geq 1, \int_{S}^{+\infty} E(t)^{2} \phi^{\prime}(t) d t \leq c E(S)
$$

Define $F(t):=E(t+1)$ and $\tilde{\phi}(t):=\phi(t+1)$ on $[0,+\infty[$. Thus we can apply the Gronwall type inequality (3.2) given by Lemma 1 with $\sigma=1$ to obtain a decay rate estimate on $F$, so on $E$ : there exists $C$ depending on $E(1)$ in a continuous way such that

$$
\forall t \geq 1, E(t) \leq \frac{C}{\phi(t)}
$$


That gives a first estimate of the decay rate of the energy. Hence the problem is to find a strictly increasing function $\phi$ satisfying the following conditions

$$
\begin{gathered}
\phi \text { is concave and } \phi(t) \longrightarrow+\infty \text { as } t \longrightarrow+\infty, \\
\phi^{\prime}(t) \longrightarrow 0 \text { as } t \longrightarrow+\infty, \\
\int_{1}^{+\infty} \phi^{\prime}(t)\left(g^{-1}\left(\phi^{\prime}(t)\right)\right)^{2} d t \text { converges, }
\end{gathered}
$$

and then to estimate the growth of $\phi$ at infinity in order to prove (2.19). If such a function exists, we can assume that $\phi(1)=1$. With the change of variable defined by

$$
\tau=\phi(t)
$$

we see that

$$
\int_{1}^{+\infty} \phi^{\prime}(t)\left(g^{-1}\left(\phi^{\prime}(t)\right)\right)^{2} d t=\int_{1}^{+\infty}\left(g^{-1}\left(\phi^{\prime}\left(\phi^{-1}(\tau)\right)\right)\right)^{2} d \tau=\int_{1}^{+\infty}\left(g^{-1}\left(\frac{1}{\left(\phi^{-1}\right)^{\prime}(\tau)}\right)\right)^{2} d \tau .
$$

Let us define $\psi$ on $[1,+\infty[$ by

$$
\forall t \geq 1, \psi(t)=1+\int_{1}^{t} \frac{1}{g\left(\frac{1}{\tau}\right)} d \tau
$$

Then $\psi$ is a strictly increasing function of class $\mathcal{C}^{2}$ on $[1,+\infty[$ that satisfies

$$
\psi^{\prime}(t)=\frac{1}{g\left(\frac{1}{t}\right)} \longrightarrow+\infty \text { as } t \longrightarrow+\infty .
$$

So

$$
\psi(t) \longrightarrow+\infty \text { as } t \longrightarrow+\infty
$$

and

$$
\int_{1}^{+\infty}\left(g^{-1}\left(\frac{1}{\psi^{\prime}(\tau)}\right)\right)^{2} d \tau=\int_{1}^{+\infty} \frac{1}{\tau^{2}} d \tau<+\infty
$$

Moreover $\psi^{\prime}$ is increasing so $\psi$ is a convex function. Then it is easy to verify that $\psi^{-1}$ is concave on $[1,+\infty[$ : derivating twice the expression

$$
\psi\left(\psi^{-1}(\tau)\right)=\tau,
$$

we see that

$$
\left(\psi^{-1}\right)^{\prime \prime}(\tau)=-\frac{\psi^{\prime \prime}\left(\psi^{-1}(\tau)\right)\left(\left(\psi^{-1}\right)^{\prime}(\tau)\right)^{2}}{\psi^{\prime}\left(\psi^{-1}(\tau)\right)}=-\frac{\psi^{\prime \prime}\left(\psi^{-1}(\tau)\right)}{\left(\psi^{\prime}\left(\psi^{-1}(\tau)\right)\right)^{3}} \leq 0
$$

That is why we define $\phi$ on $[1+\infty[$ by

$$
\forall t \geq 1, \phi(t)=\psi^{-1}(t) .
$$


Then $\phi$ is a strictly increasing concave function of class $\mathcal{C}^{2}$ on $[1+\infty[$ that satisfies all the assumptions we made in our computations: (4.32) and (4.34) are already verified (4.33) is also true because

$$
\phi^{\prime}(t)=\frac{1}{\psi^{\prime}(\phi(t))}=g\left(\frac{1}{\phi(t)}\right) \longrightarrow 0 \text { when } t \longrightarrow+\infty .
$$

(Note that $\phi(1)=1$ because $\psi(1)=1$, and that $\phi^{\prime}(1)=g(1)<1$, so it is easy to extend $\phi$ on $[0,+\infty[$ such that it remains a concave and strictly increasing nonnegative function of class $\mathcal{C}^{2}$ on $[0,+\infty[$, and such that $\phi(0)=0$.)

So we have explicitly constructed a function $\phi$ that satisfies the required properties. With that special choice we deduce from (4.29) that

$$
\begin{aligned}
\int_{S}^{T} E(t)^{2} \phi^{\prime}(t) d t & \leq c E(S)^{2}+c E(S) \int_{S}^{+\infty} \phi^{\prime}(t)\left(g^{-1}\left(\phi^{\prime}(t)\right)\right)^{2} d t \\
& \leq c E(S)^{2}+c E(S) \int_{\phi(S)}^{+\infty}\left(g^{-1}\left(\phi^{\prime}\left(\phi^{-1}(\tau)\right)\right)\right)^{2} d \tau \\
& \leq c E(S)^{2}+c E(S) \int_{\phi(S)}^{+\infty}\left(g^{-1}\left(\frac{1}{\left(\phi^{-1}\right)^{\prime}(\tau)}\right)\right)^{2} d \tau \\
& \leq c E(S)^{2}+c E(S) \int_{\phi(S)}^{+\infty} \frac{1}{\tau^{2}} d \tau=c E(S)^{2}+c \frac{E(S)}{\phi(S)}
\end{aligned}
$$

Then we can apply Lemma 3 with $\sigma=\sigma^{\prime}=1$ to deduce that

$$
\forall t \geq 1, E(t) \leq \frac{C}{\phi(t)^{2}}
$$

which is clearly a better estimate than (4.31). It remains to estimate the growth of $\phi$. This is equivalent to estimate the growth of the function $\phi^{-1}=\psi$. Set $\tau_{0}$ such that

$$
g\left(\frac{1}{\tau_{0}}\right) \leq 1
$$

By monotonicity we have

$$
\forall \tau \geq \tau_{0}, \quad \psi(\tau) \leq 1+(\tau-1) \frac{1}{g\left(\frac{1}{\tau}\right)} \leq \tau \frac{1}{g\left(\frac{1}{\tau}\right)}=\frac{1}{G\left(\frac{1}{\tau}\right)}
$$

Thus

$$
\forall \tau \geq \tau_{0}, \quad \tau \leq \phi\left(\frac{1}{G\left(\frac{1}{\tau}\right)}\right)=\phi(t) \quad \text { with } \quad t=\frac{1}{G\left(\frac{1}{\tau}\right)}
$$

so

$$
\frac{1}{\phi(t)} \leq \frac{1}{\tau}=G^{-1}\left(\frac{1}{t}\right)
$$

Thus the proof of (2.19) is achieved. 


\subsection{Second estimate on the decay rate of the energy}

Assume that $H(0)=0$ and that $H$ is nondecreasing on $[0, \eta]$ for some $\eta>0$. Set $T_{1}$ such that

$$
\forall t \geq T_{1}, H\left(\frac{1}{t}\right) \leq \eta
$$

Set $T_{2}=\sup \left\{T_{1}, \frac{1}{\eta}\right\}$. Assume that $\phi$ is an increasing and concave function such that

$$
\forall t \geq T_{2}, \phi^{\prime}(t) \leq \eta \text { and } \phi^{\prime}(t) \leq H(\eta)
$$

and also

$$
\phi^{\prime}(t) \longrightarrow 0 \text { as } t \longrightarrow+\infty
$$

Then define

$$
\forall t \geq T_{2}, \tilde{h}(t)=H^{-1}\left(\phi^{\prime}(t)\right)
$$

Since $H$ is nondecreasing on $[0, \eta], \tilde{h}$ is a nonincreasing function that satisfies

$$
\forall t \geq T_{2}, \tilde{h}(t) \leq \eta
$$

and

$$
\tilde{h}(t) \longrightarrow 0 \text { as } t \longrightarrow+\infty
$$

For every $t \geq T_{2}$, define

$$
\begin{aligned}
& \tilde{\Gamma}_{1,1}^{t}:=\left\{x \in \Gamma_{1}:\left|u^{\prime}\right| \leq \tilde{h}(t)\right\}, \\
& \tilde{\Gamma}_{1,2}^{t}:=\left\{x \in \Gamma_{1}: \tilde{h}(t)<\left|u^{\prime}\right| \leq \tilde{h}\left(T_{2}\right)\right\}, \\
& \tilde{\Gamma}_{1,3}^{t}:=\left\{x \in \Gamma_{1}:\left|u^{\prime}\right|>\tilde{h}\left(T_{2}\right)\right\} .
\end{aligned}
$$

Set $T>S \geq T_{2}$. It is easy to check that

$$
\int_{S}^{T} E \phi^{\prime} \int_{\tilde{\Gamma}_{1,3}^{t}} m \cdot \nu u^{\prime 2} d s d t \leq c \int_{S}^{T} E \phi^{\prime} \int_{\tilde{\Gamma}_{1,3}^{t}} m \cdot \nu u^{\prime} \rho\left(u^{\prime}\right) d s d t \leq c \int_{S}^{T} E\left(-E^{\prime}\right) \leq c E(S)^{2} .
$$

Since $H$ is nondecreasing on $[0, \eta]$, for all $x \in \tilde{\Gamma}_{1,2}^{t}$, we have

$$
\phi^{\prime}(t) u^{\prime 2}=H(\tilde{h}(t)) u^{\prime 2} \leq\left|H\left(u^{\prime}\right)\right| u^{\prime 2}=u^{\prime} g\left(u^{\prime}\right) \leq u^{\prime} \rho\left(u^{\prime}\right) .
$$

Thus

$$
\int_{S}^{T} E \phi^{\prime} \int_{\tilde{\Gamma}_{1,2}^{t}} m \cdot \nu u^{\prime 2} d s d t \leq \int_{S}^{T} E \int_{\tilde{\Gamma}_{1,2}^{t}} m \cdot \nu u^{\prime} \rho\left(u^{\prime}\right) d s d t \leq \frac{1}{2} E(S)^{2} .
$$


At last, we have:

$$
\int_{S}^{T} E \phi^{\prime} \int_{\tilde{\Gamma}_{1,1}^{t}} m \cdot \nu u^{\prime 2} d s d t \leq c \int_{S}^{T} E(t) \phi^{\prime}(t)\left(\int_{\tilde{\Gamma}_{1,1}^{t}} \tilde{h}(t)^{2} d s\right) d t \leq c E(S) \int_{S}^{T} \phi^{\prime}(t)\left(H^{-1}\left(\phi^{\prime}(t)\right)\right)^{2} d t .
$$

So we obtain that

$$
\forall T_{2} \leq S<T<\infty: \int_{S}^{T} E \phi^{\prime} \int_{\Gamma_{1}} m \cdot \nu u^{\prime 2} d s d t \leq c E(S)^{2}+c E(S) \int_{S}^{T} \phi^{\prime}(t)\left(H^{-1}\left(\phi^{\prime}(t)\right)\right)^{2} d t .
$$

In a similar way, for every $t \geq T_{2}$, define

$$
\begin{aligned}
& \tilde{\Gamma}_{1,4}^{t}:=\left\{x \in \Gamma_{1}: g^{-1}\left(\left|u^{\prime}\right|\right) \leq \tilde{h}(t)\right\} \\
& \tilde{\Gamma}_{1,5}^{t}:=\left\{x \in \Gamma_{1}: \tilde{h}(t)<g^{-1}\left(\left|u^{\prime}\right|\right) \leq \tilde{h}\left(T_{2}\right)\right\} \\
& \tilde{\Gamma}_{1,6}^{t}:=\left\{x \in \Gamma_{1}: g^{-1}\left(\left|u^{\prime}\right|\right)>\tilde{h}\left(T_{2}\right)\right\}
\end{aligned}
$$

Set $T>S \geq T_{2}$. Then

$$
\int_{S}^{T} E \phi^{\prime} \int_{\tilde{\Gamma}_{1,6}^{t}} m \cdot \nu \rho\left(u^{\prime}\right)^{2} d s d t \leq c \int_{S}^{T} E \phi^{\prime} \int_{\tilde{\Gamma}_{1,6}^{t}} m \cdot \nu u^{\prime} \rho\left(u^{\prime}\right) d s d t \leq c \int_{S}^{T} E\left(-E^{\prime}\right) \leq c E(S)^{2} .
$$

Since $H$ is nondecreasing on $[0, \eta]$, for all $x \in \tilde{\Gamma}_{1,5}^{t}$, we have

$$
\phi^{\prime}(t)=H(\tilde{h}(t)) \leq H\left(g^{-1}\left(\left|u^{\prime}\right|\right)\right)=\frac{\left|u^{\prime}\right|}{g^{-1}\left(\left|u^{\prime}\right|\right)} .
$$

Thus

$$
\begin{aligned}
\int_{S}^{T} E \phi^{\prime} \int_{\tilde{\Gamma}_{1,5}^{t}} m \cdot \nu \rho\left(u^{\prime}\right)^{2} d s d t & \leq \int_{S}^{T} E \int_{\tilde{\Gamma}_{1,5}^{t}} m \cdot \nu \frac{\left|u^{\prime}\right|}{g^{-1}\left(\left|u^{\prime}\right|\right)} \rho\left(u^{\prime}\right)^{2} d s d t \\
& \leq \int_{S}^{T} E \int_{\tilde{\Gamma}_{1,5}^{t}} m \cdot \nu u^{\prime} \rho\left(u^{\prime}\right) d s d t \leq \frac{1}{2} E(S)^{2}
\end{aligned}
$$

At last, we have:

$$
\begin{aligned}
\int_{S}^{T} E \phi^{\prime} \int_{\tilde{\Gamma}_{1,4}^{t}} m \cdot \nu \rho\left(u^{\prime}\right)^{2} d s d t & \leq c \int_{S}^{T} E \phi^{\prime}\left(\int_{\tilde{\Gamma}_{1,4}^{t}} g^{-1}\left(\left|u^{\prime}\right|\right)^{2} d s\right) d t \\
& \leq c E(S) \int_{S}^{T} \phi^{\prime}(t)\left(H^{-1}\left(\phi^{\prime}(t)\right)\right)^{2} d t
\end{aligned}
$$

So we obtain that

$$
\forall T_{2} \leq S<T<\infty: \int_{S}^{T} E \phi^{\prime} \int_{\Gamma_{1}} m \cdot \nu \rho\left(u^{\prime}\right)^{2} d s d t \leq c E(S)^{2}+c E(S) \int_{S}^{T} \phi^{\prime}(t)\left(H^{-1}\left(\phi^{\prime}(t)\right)\right)^{2} d t .
$$

(Note that $c$ does not depend on the constant of Lipschitz of $\rho$.) 
So define

$$
\forall t \geq T_{2}, \tilde{\phi}^{-1}(t)=T_{2}+\int_{T_{2}}^{t} \frac{1}{H\left(\frac{1}{\tau}\right)} d \tau
$$

Then

$$
\forall t \geq T_{2}, \tilde{\phi}(t) \geq T_{2} \geq \frac{1}{\eta}
$$

so

$$
\forall t \geq T_{2}, \tilde{\phi}^{\prime}(t)=H\left(\frac{1}{\tilde{\phi}(t)}\right) \leq H(\eta)
$$

and

$$
\forall t \geq T_{2}, \tilde{\phi}^{\prime}(t) \leq \tilde{\phi}^{\prime}\left(T_{2}\right)=H\left(\frac{1}{\phi\left(T_{2}\right)}\right)=H\left(\frac{1}{T_{2}}\right) \leq H\left(\frac{1}{T_{1}}\right) \leq \eta
$$

Then $\tilde{\phi}$ satisfies all the required properties. Since

$$
\forall y \in[0,1], g(y) \leq y \text { i.e. } H(y) \leq 1,
$$

we see that

$$
\tilde{\phi}^{-1}(t) \leq T_{2}+\frac{t-T_{2}}{H\left(\frac{1}{t}\right)} \leq \frac{t}{H\left(\frac{1}{t}\right)}=\frac{1}{g\left(\frac{1}{t}\right)}
$$

Thus we deduce that

$$
\forall t \geq T_{2}, E(t) \leq C \frac{1}{\tilde{\phi}(t)^{2}} \leq C\left(g^{-1}\left(\frac{1}{t}\right)\right)^{2}
$$

So the proof of Theorem 2 is completed when $\rho$ is globally Lipschitz.

\subsection{The case of the polynomial behavior}

If $g(y)=y^{p}$ for some $p>1$ on $(0,1)$, then $H(y)=y^{p-1}$ is increasing on $(0,1)$. So $(2.20)$ gives the estimate

$$
E(t) \leq \frac{C}{t^{2 / p}} .
$$

We did not manage to find a function $\phi$ that gives directly the estimate found by Zuazua [35]:

$$
E(t) \leq \frac{C}{t^{2 /(p-1)}}
$$

However the method leading to (2.20) allows us to prove this estimate: set $n \geq 1, \lambda_{n}>0$ and define

$$
\forall t \geq 1, \phi_{n}(t)=t^{1 /(1+n(p-1))} \text { and } h_{n}(t)=\left(\lambda_{n} \phi_{n}^{\prime}(t)\right)^{1 /(p-1)} .
$$


We see that $\phi_{n}$ is a concave function that satisfies (4.11). So we derive from (4.4) that $E$ satisfies for all $S \geq 1$ :

$$
\begin{aligned}
\int_{S}^{+\infty} E(t)^{2} \phi_{n}^{\prime}(t) d t & \leq c \phi_{n}^{\prime}(S) E(S)^{2}+c E(S) \int_{S}^{+\infty} \phi_{n}^{\prime}(t) h_{n}(t)^{2} d t \\
& \leq c\left(\frac{1}{\lambda_{n}}+\phi_{n}^{\prime}(S)\right) E(S)^{2}+\lambda_{n}^{2 /(p-1)} n^{-(p+1) /(p-1)} \frac{E(S)}{\phi_{n}(S)^{2 n-1}}
\end{aligned}
$$

where $c$ denotes a positive constant that does not depend on $n$. We minimize the value of the right-hand side term with respect to $\lambda_{n}$ to get that

$$
\int_{S}^{+\infty} E(t)^{2} \phi_{n}^{\prime}(t) d t \leq \frac{c}{n} \phi_{n}(S)^{-\frac{p-1}{p+1}(2 n-1)} E(S)^{1+\frac{2}{p+1}} .
$$

Then we apply Corollary 3.1 to the function $f$ defined by the right-hand side term of (4.58) and after some computations we get that

$$
\forall t \geq 1, \forall n \geq 1, E(t) \leq C_{p} t^{-\alpha_{n}}
$$

where $C_{p}$ is a positive constant that does not depend on $n$, and

$$
\alpha_{n}:=\frac{2 n+(2 /(p-1))}{1+n(p-1)} \longrightarrow \frac{2}{p-1} \text { as } n \rightarrow+\infty .
$$

Hence letting go $n$ to infinity, we get (2.16).

\section{Decay RAte estimate When $\rho$ IS NOT SUPPOSED TO BE GlOBAlly Lipschitz}

In Section 3, we proved Theorem 2 in the case where $\rho$ is globally Lipschitz. In fact, we used this additional property only to justify our computations, thanks to the regularity provided by (2.12-2.13). Note that the constant of Lipschitz of $\rho$ never appeared in our estimates. We will remove the additional hypothesis on $\rho$ in the following way: first we construct a sequence of globally Lipschitz functions $\rho_{k}$ that converges to $\rho$; then we apply a general perturbation theorem to prove that the sequence $\left(u_{k}\right)_{k \in \mathbb{N}}$ of the solution of the problem (2.1-2.4) where $\rho_{k}$ replaces $\rho$ converges to the solution $u$ of $(2.1-2.4)$.

First we construct a sequence of globally Lipschitz functions $\rho_{k}$ that converges to $\rho$ :

Lemma 9. Let $\rho: \mathbb{R} \longrightarrow \mathbb{R}$ be a nondecreasing, continuous function satisfying (2.7). Then there exists a sequence of nondecreasing, globally Lipschitz, continuous functions $\rho_{k}: \mathbb{R} \longrightarrow \mathbb{R}$ satisfying for all $k \in \mathbb{N} \backslash\{0\}$ :

$$
\left\{\begin{array}{l}
\forall y \in[-1,1],\left|g\left(\frac{y}{2}\right)\right| \leq\left|\rho_{k}(y)\right| \leq\left|g^{-1}(y)\right|, \\
\forall|y| \geq 1, c_{1}^{\prime}|y| \leq\left|\rho_{k}(y)\right| \leq c_{2}^{\prime}|y|
\end{array}\right.
$$

with suitables constants $c_{1}^{\prime}$ and $c_{2}^{\prime}$ that are independent of $k$, and such that

$$
\forall y \in \mathbb{R},\left|\rho_{k}(y)\right| \leq|\rho(y)|,
$$

and

$$
\forall y \in \mathbb{R}, \rho_{k}(y) \longrightarrow \rho(y) \text { as } k \longrightarrow+\infty .
$$


Remark. Define $g_{0}(y)=g\left(\frac{y}{2}\right)$ for all $y \in[-1,1]$; then $g_{0}^{-1}(y)=2 g^{-1}(y)$. So the functions $\rho_{k}$ and $\rho$ satisfy:

$$
\left\{\begin{array}{l}
\forall y \in[-1,1],\left|g_{0}(y)\right| \leq\left|\rho_{k}(y)\right| \leq\left|g_{0}^{-1}(y)\right|, \\
\forall|y| \geq 1, c_{1}^{\prime}|y| \leq\left|\rho_{k}(y)\right| \leq c_{2}^{\prime}|y| .
\end{array}\right.
$$

Since $\rho_{k}$ is globally Lipschitz, we can apply the results of Section 3 to the the solution $u_{k}$ of the problem

$$
\left\{\begin{array}{l}
u_{k}^{\prime \prime}-\Delta u_{k}=0 \text { in } \Omega \times \mathbb{R}_{+} \\
u_{k}=0 \text { on } \Gamma_{0} \times \mathbb{R}_{+} \\
\partial_{\nu} u_{k}+m \cdot \nu \rho_{k}\left(u_{k}^{\prime}\right)=0 \text { on } \Gamma_{1} \times \mathbb{R}_{+} \\
u_{k}(0)=u^{0}, u_{k}^{\prime}(0)=u^{1}
\end{array}\right.
$$

The energy $E\left(u_{k}\right)$ of $u_{k}$ satisfies the following estimate

$$
\forall t \geq 1, E\left(u_{k}\right)(t) \leq c\left(G_{0}^{-1}\left(\frac{1}{t}\right)\right)^{2},
$$

where $c$ is a constant that depends on $E(0)$ (in a continuous way) but that does not depend on $k$. Since

$$
\forall y \geq 0, G_{0}^{-1}(y)=2 G^{-1}\left(\frac{y}{2}\right) \leq 2 G^{-1}(y),
$$

we obtain that

$$
\forall t \geq 1, E\left(u_{k}\right)(t) \leq 4 c\left(G^{-1}\left(\frac{1}{t}\right)\right)^{2}
$$

Thus, if we prove that

$$
\forall t \geq 1, E\left(u_{k}\right)(t) \longrightarrow E(u)(t) \text { as } k \longrightarrow+\infty
$$

the proof of Theorem 2 will be completed.

Proof of Lemma 9. Set $k \geq 1$ and define:

$$
\forall y \in \mathbb{R}, \rho_{k}(y)=\rho\left(\left(\operatorname{id}_{\mathbb{R}}+\frac{1}{k} \rho\right)^{-1}(y)\right)
$$

that means:

$$
\forall y \in \mathbb{R}, \rho_{k}(y)=\rho\left(y_{k}\right),
$$

where $y_{k}$ is the unique real number that satisfy

$$
y_{k}+\frac{1}{k} \rho\left(y_{k}\right)=y
$$

One may readily verify that the functions $\rho_{k}$ are well-defined, continuous and nondecreasing. They are also globally Lipschitz: set $y^{1}, y^{2}$ in $\mathbb{R}$ and denote $y_{k}^{1}$ and $y_{k}^{2}$ the real numbers that satisfy (5.10): then

$$
y_{k}^{1}-y_{k}^{2}+\frac{1}{k}\left(\rho\left(y_{k}^{1}\right)-\rho\left(y_{k}^{2}\right)\right)=y^{1}-y^{2} ;
$$


using the monotonicity of $\operatorname{id}_{\mathbb{R}}+\frac{1}{k} \rho$ and of $\rho$, we obtain that $y^{1}-y^{2}, y_{k}^{1}-y_{k}^{2}$ and $\rho\left(y_{k}^{1}\right)-\rho\left(y_{k}^{2}\right)$ have the same sign, and so

$$
\left|\rho\left(y_{k}^{1}\right)-\rho\left(y_{k}^{2}\right)\right| \leq k\left|y^{1}-y^{2}\right|
$$

Next we prove that (5.2) and (5.3) are satisfied on $[0,+\infty$ [ (the study is similar on $]-\infty, 0]$ ). Set $y \geq 0$. If $y=0$, then $y_{k}=0$. If $y>0$, then $y_{k}>0, \rho\left(y_{k}\right)>0$, and so $y_{k} \leq y$. That implies that (5.2) is satisfied:

$$
\rho_{k}(y)=\rho\left(y_{k}\right) \leq \rho(y) .
$$

It is also clear that the sequence $\left(y_{k}\right)_{k \geq 1}$ is nondecreasing:

$$
y_{k+1}-y_{k}=\frac{1}{k} \rho\left(y_{k}\right)-\frac{1}{k+1} \rho\left(y_{k+1}\right),
$$

and if $y_{k+1}<y_{k}$, then

$$
y_{k+1}-y_{k} \geq \frac{1}{k} \rho\left(y_{k}\right)-\frac{1}{k+1} \rho\left(y_{k}\right)>0 .
$$

Then the sequence $\left(y_{k}\right)_{k \geq 1}$ converges, and it is easy to see that

$$
y_{k} \longrightarrow y \text { as } k \longrightarrow+\infty,
$$

so (5.3) is satisfied:

$$
\rho_{k}(y)=\rho\left(y_{k}\right) \longrightarrow \rho(y) \text { as } k \longrightarrow+\infty .
$$

At last we prove that (5.1) is satisfied: we deduce from (5.2) that

$$
\left\{\begin{array}{l}
\forall y \in[0,1], \rho_{k}(y) \leq \rho(y) \leq g^{-1}(y), \\
\forall y \geq 1, \rho_{k}(y) \leq \rho(y) \leq c_{2} y
\end{array}\right.
$$

On the other hand

$$
\text { if } y_{k} \leq \frac{y}{2}, \text { then } \rho_{k}(y)=\rho\left(y_{k}\right) \geq \frac{k}{2} y \geq \frac{y}{2}
$$

and in the other case,

$$
\text { if } y_{k} \geq \frac{y}{2} \text {, then } \rho_{k}(y)=\rho\left(y_{k}\right) \geq \rho\left(\frac{y}{2}\right) \text {. }
$$

So

$$
\text { if } y \in[0,1], \text { then } \rho_{k}(y) \geq \inf \left\{\rho\left(\frac{y}{2}\right), \frac{y}{2}\right\} \geq g\left(\frac{y}{2}\right)
$$

(we used the fact that for all $y \in[0,1], g(y) \leq g^{-1}(y)$ so $g(y) \leq y$ ); and similarly we deduce that

$$
\text { if } y \geq 1 \text {, then } \rho_{k}(y) \geq \inf \left\{\rho\left(\frac{y}{2}\right), \frac{y}{2}\right\} \geq c y
$$

with some $c>0$ (we use (2.7) for $y \in\left[2,+\infty\left[\right.\right.$ and the continuity of $\rho$ on $\left[\frac{1}{2}, 1\right]$ ).

It remains to prove (5.7). This is a consequence of the following perturbation theorem (see, e.g., Barbu [2] Prop. 4.2.1 and Th. 4.2.1): 
Theorem 3. Let $\mathcal{A}$ and $\mathcal{A}_{k}(k \in \mathbb{N})$ be maximal monotone operators in a Hilbert space and assume that

$$
\left(I+\mathcal{A}_{k}\right)^{-1} W \longrightarrow(I+\mathcal{A})^{-1} W \text { in } \mathcal{H}
$$

for every $W \in \mathcal{H}$ as $k \longrightarrow+\infty$. Choose $U^{0}, U_{k}^{0} \in \mathcal{H}$ such that

$$
U_{k}^{0} \longrightarrow U^{0} \text { in } \mathcal{H}
$$

Then the corresponding solutions of

$$
U^{\prime}+\mathcal{A} U=0 \text { in } \mathbb{R}_{+}, \quad U(0)=U^{0}
$$

and

$$
U_{k}^{\prime}+\mathcal{A}_{k} U_{k}=0 \text { in } \mathbb{R}_{+}, \quad U_{k}(0)=U_{k}^{0}
$$

satisfy

$$
U_{k}(t) \longrightarrow U(t) \text { in } \mathcal{H} \text { as } k \longrightarrow+\infty
$$

for every $t \in \mathbb{R}_{+}$.

Admit that Theorem 3 can be applied: then (5.7) is a consequence of (5.12). It remains to show that we can apply Theorem 3 so we need to prove that

$$
\left(I+\mathcal{A}_{k}\right)^{-1} W \longrightarrow(I+\mathcal{A})^{-1} W \text { in } \mathcal{H}
$$

where $\mathcal{A}_{k}$ denotes the generator of the semigroup associated with the problem (5.5). It is not difficult to see that this is a consequence of (5.3) and of the following estimate provided by (5.1): there exists a positive constant $c$ such that all the functions $\rho_{k}$ (and also the function $\rho$ ) satisfy:

$$
\forall k \in \mathbb{N} \backslash\{0\}, \forall y \in \mathbb{R},\left|\rho_{k}(y)\right| \leq c(1+|y|)
$$

(for more details, see, e.g., Komornik [18] p. 132).

The author thanks the referees and E. Zuazua for their valuable suggestions concerning the presentation of our results.

\section{REFERENCES}

[1] M. Aassila, On a quasilinear wave equation with a strong damping. Funkcial. Ekvac. 41 (1998) 67-78.

[2] V. Barbu, Analysis and control of nonlinear infinite dimensional systems. Academic Press, New York (1993).

[3] C. Bardos, G. Lebeau and J. Rauch, Sharp sufficient conditions for the observation, control and stabilization of waves from the boundary. SIAM J. Control Optim. 30 (1992) 1024-1065.

[4] A. Carpio, Sharp estimates of the energy for the solutions of some dissipative second order evolution equations. Potential Anal. 1 (1992) 265-289.

[5] G. Chen, Energy decay estimates and exact boundary value controllability for the wave equation in a bounded domain. $J$. Math. Pures Appl. 58 (1979) 249-274.

[6] G. Chen and H. Wang, Asymptotic behavior of solutions of the one dimensional wave equation with a nonlinear boundary stabilizer. SIAM J. Control Optim. 27 (1989) 758-775.

[7] F. Chentouh, Décroissance de l'énergie pour certaines équations hyperboliques semilinéaires dissipatives. Thèse de $3^{\mathrm{e}}$ cycle, Université Pierre et Marie Curie(1984).

[8] F. Conrad, J. Leblond and J. P. Marmorat, Stabilization of second order evolution equations by unbounded nonlinear feedback in. Proc. of the Fifth IFAC Symposium on Control of Distributed Parameter Systems, Perpignan (1989) 101-116. 
[9] F. Conrad and B. Rao, Decay of solutions of wave equations in a star-shaped domain with non-linear boundary feedback. Asymptotic Analysis 7 (1993) 159-177.

[10] C.M. Dafermos, Asymptotic behavior of solutions of evolutions equations, Nonlinear evolution equations, M.G. Crandall, Ed., Academic Press, New-York (1978) 103-123.

[11] A. Haraux, Comportement à l'infini pour une équation des ondes non linéaire dissipative. C. R. Acad. Sci. Paris Sér. A 287 (1978) 507-509.

[12] A. Haraux, Oscillations forcées pour certains systèmes dissipatifs non linéaires. Publication du Laboratoire d'Analyse Numérique No. 78010, Université Pierre et Marie Curie, Paris (1978).

[13] A. Haraux and E. Zuazua, Decay estimates for some semilinear damped hyperbolic problems. Arch. Rat. Mech. Anal. 100 (1988) 191-206.

[14] M.A. Horn and I. Lasiecka, Global stabilization of a dynamic Von Karman plate with nonlinear boundary feedback. Appl. Math. Optim. 31 (1995) 57-84.

[15] M.A. Horn and I. Lasiecka, Nonlinear boundary stabilization of parallelly connected Kirchhoff plates. Dynamics and Control 6 (1996) 263-292.

[16] V. Komornik and E. Zuazua, A direct method for the boundary stabilization of the wave equation. J. Maths Pures Appl. 69 (1990) 33-54.

[17] V. Komornik, On the nonlinear boundary stabilization of the wave equation. Chinese Ann. Math. Ser. B. 14 (1993) 153-164.

[18] V. Komornik, Exact Controllability and Stabilization. RAM: Research in Applied Mathematics. Masson, Paris; John Wiley, Ltd., Chichester (1994).

[19] S. Kouémou Patcheu, On the decay of solutions of some semilinear hyperbolic problems. Panamer. Math. J. 6 (1996) 69-82.

[20] J.E. Lagnese, Decay of solutions of wave equations in a bounded region with boundary dissipation. J. Differential Equations 50 (1983) 163-182.

[21] J.E. Lagnese, Boundary stabilization of thin plates. SIAM Studies in Appl. Math., Philadelphia, 1989.

[22] I. Lasiecka and D. Tataru, Uniform boundary stabilization of semilinear wave equations with nonlinear boundary damping. $J$. Diff. Integr. Eq. 6 (1993) 507-533.

[23] I. Lasiecka, Uniform stabilizability of a full Von Karman system with nonlinear boundary feedback. SIAM J. Control Optim. 36 (1998) 1376-1422.

[24] I. Lasiecka, Boundary stabilization of a 3-dimensional structural acoustic model. J. Math. Pures Appl. 78 (1999) $203-232$.

[25] J.L. Lions, Contrôlabilité exacte et stabilisation de systèmes distribués, Vol. 1, Masson, Paris (1988).

[26] W.-J. Liu and E. Zuazua, Decay rates for dissipative wave equation, preprint.

[27] P. Martinez, Decay of solutions of the wave equation with a local highly degenerate dissipation. Asymptotic Analysis 19 (1999) $1-17$.

[28] P. Martinez, A new method to obtain decay rate estimates for dissipative systems with localized damping. Rev. Mat. Compl Madrid, to appear.

[29] M. Nakao, Asymptotic stability of the bounded or almost periodic solution of the wave equation with a nonlinear dissipative term. J. Math. Anal. Appl. 58 (1977) 336-343.

[30] M. Nakao, Decay of solutions of the wave equation with a local nonlinear dissipation. Math. Ann. 305 (1996) $403-417$.

[31] L.R. Tcheugoué Tébou, Stabilization of the wave equation with localized nonlinear damping. J. Differential Equations 145 (1998) 502-524.

[32] J. Vancostenoble, Optimalité d'estimations d'énergie pour une équation des ondes amortie. C. R. Acad. Sci. Paris Sér. A, to appear.

[33] J. Vancostenoble and P. Martinez, Optimality of energy estimates for a damped wave equation with polynomial or non polynomial feedbacks, submitted.

[34] E. Zuazua, Stability and decay for a class of nonlinear hyperbolic problems. Asymptotic Analysis 1 (1988) 1-28.

[35] E. Zuazua, Uniform stabilization of the wave equation by nonlinear boundary feedback. SIAM J. Control and Optim. 28 (1990) 466-478. 